\title{
Tumor neuroendócrino primário de mama: relato de três casos e revisão de literatura
}

\section{Primary neuroendocrine carcinoma of the breast: case report and literature review}

Mariana Novaes Pinheiro'; Jurandyr Moreira de Andrade'; Liane Rapatoni ${ }^{3}$; Alfredo Ribeiro Silva ${ }^{4}$; Daniel Guimarães Tiezzi ${ }^{5}$; Hélio Humberto Angotti Carrara ${ }^{6}$; Fábio Eduardo Zola7; Harley Francisco de Oliveira ${ }^{8}$; Fernanda Maris Peria

unitermos
Câncer de mama
Carcinoma neuroendócrino
Achado clinicopatológico
Análise imuno-
histoquímica

\section{resumo}

Os tumores neuroendócrinos primários de mama (TNPMs) são incomuns e não há consenso quanto a tratamento e prognóstico. No presente trabalho, foram revisados os diagnósticos de 1.184 pacientes com câncer de mama atendidos no Hospital das Clínicas da Faculdade de Medicina de Ribeirão Preto da Universidade de São Paulo (HCFMRP/USP), identificando três casos que preenchiam os critérios de TNPM, segundo classificação estabelecida pela Organização Mundial da Saúde (OMS) em 2003. Foram avaliados os achados clinicopatológicos e imuno-histoquímicos e as terapias realizadas, buscando caracterizar os padrões histopatológicos e de comportamento distintos dos carcinomas convencionais de mama.

\section{abstract}

Primary neuroendocrine breast carcinomas (NECS) are uncommon. Moreover, there is no consensus as to its treatment and prognosis. In this study, the diagnoses of 1,184 cases of breast cancer treated at Hospital das Clínicas da Faculdade de Medicina de Ribeirão Preto/Universidade de São Paulo (HCFMRP/USP) were reviewed. Three among them fulfilled the criteria for primary NEC according to the classification established by the World Health Organization (WHO) in 2003. Clinicopathological, immunohistochemical features and treatments were assessed in order to characterize histopathological and distinct patterns of conventional breast carcinomas. key words

Breast cancer

Neuroendocrine

carcinoma

Clinicopathological study

Immunohistochemistry

\footnotetext{
1. Oncologista clínica formada pelo Hospital das Clínicas da Faculdade de Medicina de Ribeirão Preto da Universidade de São Paulo (HCFMRP/USP).

2. Professor titular do Departamento de Cinecologia e Obstetrícia da FMRP/USP.

3. Médica assistente da Divisão de Oncologia de Oncologia Clínica do HCFMRP/USP.

4. Professor do Departamento de Patologia e Medicina Legal da FMRP/USP.

5. Professor do Departamento de Ginecologia e Obstetrícia da FMRP/USP.

6. Professor do Departamento de Cinecologia e Obstetrícia da FMRP/USP.

7. Médico assistente da Divisão de Oncologia de Oncologia Clínica do HCFMRP/USP.

8. Professor do Departamento de Clínica Médica da FMRP/USP.

9. Professora do Departamento de Clínica Médica da FMRP/USP.
} 


\section{Introdução}

Os tumores neuroendócrinos primários de mama (TNPMs) representam aproximadamente $2 \%$ a $5 \%$ dos tumores da mama ${ }^{(20)}$. Inicialmente descritos em 1963, somente em 2003 a Organização Mundial da Saúde (OMS) definiu a necessidade de expressão de marcadores neuroendócrinos em mais de $50 \%$ das células tumorais para o diagnóstico definitivo de TNPM ${ }^{(20)}$. Os subtipos histológicos identificados foram sólido, de pequenas células, de grandes células, celular mucinoso, carcinoide atípico e carcinoma ductal neuroendócrino in situ (NE-DCIS) ${ }^{(20)}$. Alguns carcinomas de mama expressam áreas de diferenciação neuroendócrina, $<50 \%$ das células, porém sem impacto no prognóstico ${ }^{(12,21)}$.

As características histológicas dos TNPMs são semelhantes às encontradas nos tumores neuroendócrinos do trato gastrointestinal e pulmonares, sendo obrigatório investigar e excluir o tumor primário extramamário e demonstrar um componente in situ na mama ${ }^{(5,8)}$.

Não há tratamento padrão estabelecido. Apesar de relatos sugerirem comportamento agressivo, o prognóstico em estágios precoces e lesões in situ pode ser melhor que no do carcinoma ductal de mama ${ }^{(6,7,22)}$.

Em revisão da literatura de 2010, Wei et al. documentaram apenas cinco séries de casos classificadas segundo os critérios diagnósticos da OMS, a maior delas com 74 pacientes ${ }^{(4,10,11,14,17,22,25)}$.

No presente estudo, foram revisados os diagnósticos de 1.184 casos de câncer de mama atendidos no Hospital das Clínicas da Faculdade de Medicina de Ribeirão Preto da Universidade de São Paulo (HCFRMP/USP), identificando três casos que preenchiam os critérios de TNPM. Foram avaliados os achados clinicopatológicos e imuno-histoquímicos e as terapias realizadas, buscando caracterizar padrões histopatológicos e de comportamento distintos dos carcinomas convencionais de mama.

\section{Relato dos casos}

\section{Caso 1}

Mulher de 59 anos de idade com queixa de descarga papilar. O exame físico evidenciava dois nódulos palpáveis no quadrante superior medial (QSM) da mama direita, medindo $1,2 \mathrm{~cm}$ cada, sem linfonodos palpáveis. A mamografia apresentou assimetria focal em QSM e microcalcificações esparsas. Exames de estadiamento sem evidências de doença sistêmica.
Diagnosticou-se carcinoma neuroendócrino intraductal na biópsia excisional, seguida de controle local, com mastectomia associada à biópsia de linfonodo sentinela. Foram identificados na histologia múltiplos focos residuais de carcinoma papilífero in situ, variante sólida neuroendócrina medindo $1,5 \mathrm{~cm}$, moderadamente diferenciado e sem comprometimento em amostra linfonodal. $\mathrm{O}$ estádio clínico $(\mathrm{EC})$ foi Tis $\mathrm{NOMO}=0^{(2)}$. Realizou-se tamoxifeno; paciente permaneceu sem evidência de doença, com seguimento de 39 meses.

\section{Caso 2}

Mulher de 67 anos de idade com nódulo em QSM da mama esquerda medindo $3,6 \mathrm{~cm}$, sem linfonodos palpáveis. A mamografia evidenciou nódulo parcialmente circunscrito, medindo $4 \mathrm{~cm}$. Exames de estadiamento sem evidências de doença sistêmica. A punção biópsia aspirativa sugeria malignidade. Foi submetida à mastectomia com biópsia de linfonodo sentinela, identificando carcinoma neuroendócrino invasivo, moderadamente diferenciado, medindo $4 \mathrm{~cm}$, sem metástase linfonodal. EC: T2NOM0 $=\| A^{(2)}$. Realizou-se quimioterapia (seis ciclos de 5fluoracil, epirrubicina e ciclofosfamida [FEC75]), seguida do uso de tamoxifeno adjuvante. Após 24 meses do diagnóstico inicial, apresentou recidiva em plastrão e axila esquerda, submetendo-se ao esvaziamento axilar, encontrando seis gânglios acometidos. Em seguida, foi realizada radioterapia de plastrão e fossa supraclavicular e feito o uso de letrozol, por 14 meses, até o óbito decorrente de causa não relacionada ao câncer, com sobrevida global de 38 meses.

\section{Caso 3}

Mulher de 45 anos de idade, com nódulo de $5 \mathrm{~cm}$ entre os quadrantes externos da mama direita, sem linfonodos palpáveis. Exames de estadiamento sem evidências de doença sistêmica. Realizaram-se dois ciclos de quimioterapia neoadjuvante (esquema 5fluoracil, adriamicina e ciclofosfamida [FAC]) e mastectomia com esvaziamento axilar à direita. Diagnosticado tumor neuroendócrino, moderadamente diferenciado, medindo $3 \mathrm{~cm}$ com 5 linfonodos acometidos pelo neoplasia de 40 avaliados. EC: T2N2M0 $=$ IIIA (2). Devido a comorbidades graves, foram realizadas quimioterapia adjuvante com a combinação de ciclofosfamida, metotrexate e fluoracil (CMF) (seis ciclos) e radioterapia em plastrão e fossa supraclavicular, seguidas de hormonioterapia com tamoxifeno por cinco anos. Onze anos após o diagnóstico, apresentou metástases pleurais/ósseas, tratadas com paclitaxel paliativo seguido de letrozol. Foi realizada radioterapia 
antiálgica nos arcos costais e no ombro esquerdo e feito o uso de ácido zoledrônico. Após progressão de doença óssea, foi introduzido exemestano. Devido a sucessivas progressões, pulmonares, hepáticas e do sistema nervoso central (SNC), outros esquemas de quimioterapia foram utilizados, como capecitabina e gencitabina + cisplatina. Com sobrevida global de 169 meses, evoluiu para óbito devido à neoplasia.

\section{Histologia e imuno-histoquímica}

Foram revisados os diagnósticos de 1.184 casos de câncer de mama, tratados no HCFMRP/USP, entre $1^{\circ}$ de janeiro 2000 e 31 de julho de 2010, separados os casos morfologicamente compatíveis com TNPM e reclassificados como tal, segundo os critérios da OMS de 2003. A avaliação incluiu outros marcadores relacionados, como receptor para estrógeno (RE), receptor para progesterona (RP), receptor tipo 2 do fator de crescimento epidérmico humano (HER2), antígeno carcinoembrionário (CEA), citoqueratinas 7 e 20 (CK7, CK20), calponina e Ki-67 (Tabela).

\section{Discussão}

Os TNPMs foram inicialmente descritos há mais de 40 anos, mas os critérios precisos para diagnóstico histológico foram determinados apenas em 2003, segundo a classificação da $\mathrm{OMS}^{(20)}$, justificando a falta de uniformidade quanto ao diagnóstico e ao tratamento encontrados em diversos trabalhos publicados.
Diferente dos subtipos histológicos especiais de carcinomas de mama (tubular, mucinoso e cribiforme), os TNPMs não apresentam comportamento biológico bem caracterizado. Alguns estudos sugerem melhor prognósti$\mathrm{CO}^{(10,14,25)}$, outros não demonstram diferença ${ }^{(11,17)}$ e alguns ainda caracterizam pior prognóstico ${ }^{(22)}$. Em recente estudo, Weigelt et al. demonstraram que o carcinoma celular mucinoso, variante do TNPM, possui características moleculares distintas do carcinoma ductal(23). O carcinoma de pequenas células, outra variante do TNPM, demonstra prognóstico muito pior que outras histologias $(12,15,16,24)$.

O NE-DCIS foi descrito pela primeira vez em $1985^{(3)}$. Kawasaki et al. realizaram estudo de caso-controle avaliando 294 casos de carcinoma ductal in situ (DCIS); destes, 6,8\% eram NE-DCIS (7). Concluiu-se que NE-DCIS apresenta menor grau nuclear e na classificação de Van Nuys, maior escore de expressão de RE e RP e menor expressão de HER2 que os não NE-DCIS(6,7).

Os TNPMs subtipo sólido caracterizam-se histologicamente pela disposição das células tumorais em ilhas sólidas, separadas por estroma fibrovascular. Ocasionalmente, observa-se arranjo polarizado das células tumorais pelo lúmen, assemelhando-se a rosetas, com grânulos eosinofílicos e tendência a formar paliçadas periféricas ou ilhas tissulares em um clássico padrão carcinoide-like ${ }^{(10)}$ (Figura 1).

Nos três casos descritos, duas pacientes apresentavam menos de 60 anos de idade ao diagnóstico. Todas com tumores de diâmetro inferior a $5 \mathrm{~cm}$, axila negativa ao exame clínico e ausência de metástases, configurando estádios clínicos iniciais. O estádio inicial aliado a outros fatores

\begin{tabular}{ll|l|l}
\cline { 2 - 3 } Tabela & \multicolumn{2}{l}{ Perfil imuno-histoquímico para TNPM } & \\
\hline & Caso 1 & Caso 2 & Caso3 \\
\hline RE & Positivo & Positivo & Positivo \\
\hline RP & Positivo & Negativo & Positivo \\
\hline HER2 & Negativo & Negativo & Negativo \\
\hline Sinaptofisina & Positivo (90\%) & Positivo (difusa) & Negativo \\
\hline Cromogranina & Positivo (40\%) & Negativo & Negativo \\
\hline Enolase neurônio-específica & Positivo (difusa) & Positivo (difusa) & Positivo (difusa) \\
\hline Ki-67 & $<2 \%$ & $>20 \%$ & $<1 \%$ \\
\hline Calponina & Positivo & Negativo & Negativo \\
\hline CEA & Positivo (difusa) & Positivo (focal) & Positivo (focal) \\
\hline CK7 & Positivo (focal) & Positivo (focal) & Positivo (difusa) \\
\hline CK20 & Negativo & Negativo & Negativo \\
\hline
\end{tabular}

TNPM: tumor neuroendócrino primário de mama; RE: receptor para estrógeno; RP: receptor para progesterona; HER2: receptor do tipo 2 do fator de crescimento epidérmico humano ; CEA: antígeno carcinoembrionário; CK: citoqueratina. 
prognósticos, como grau de diferenciação, expressão de receptores hormonais e HER2, e o fato de tratar-se de variante subtipo sólido dos TNPMs (melhor evolução) favoreceram melhor evolução clínica dos casos. Outro critério não encontrado nesses casos, mas correlacionado com melhor prognóstico nos TNPMs, foi a diferenciação mucinosa ${ }^{(18,22)}$.

Nos casos apresentados, houve $100 \%$ de positividade para enolase neurônio-específica (NSE), positividade difusa para sinaptofisina (SNF) em dois casos, além de positividade moderada para cromogranina (CRM) em um caso, confirmando o diagnóstico de TNPM (Figuras 2 e 3).

Os três casos apresentavam componente intraductal, positividade para CK7 e negatividade para CK20, caracterizando o perfil imuno-histoquímico dos carcinomas mamários e reforçando a mama como sítio primário tumoral $^{(1)}$. Eles expressavam RE e RP, com exceção do caso 2, negativo para RP, e todos foram negativos para HER2, em concordância com a literatura. Extrapolando os achados clínico-histopatológicos, poderíamos inferir que eles pertencem ao subtipo luminal $A$ dos carcinomas de mama ${ }^{(10,19,22) .}$ Houve, também, baixa expressão de Ki-67 em dois casos (1 e 3), demonstrando baixa proliferação celular e grau moderado de diferenciação celular em todos os casos, compatíveis com comportamento clínico menos agressivo.

Quanto ao tratamento realizado, seguiu-se o protocolo para carcinomas invasivos, incluindo cirurgia, radioterapia, quimioterapia e hormonioterapia. Essa abordagem está consoante com a literatura, com exceção de alguns relatos de casos de tumores de pequenas células, tratados com quimioterapia, contendo etoposide e cisplatina ${ }^{(1,9)}$. Não se definiu tratamento específico padrão que cause impacto clinicamente significativo na sobrevida global(22).

As três pacientes foram submetidas à mastectomia e à pesquisa de linfonodo sentinela (dois casos) ou

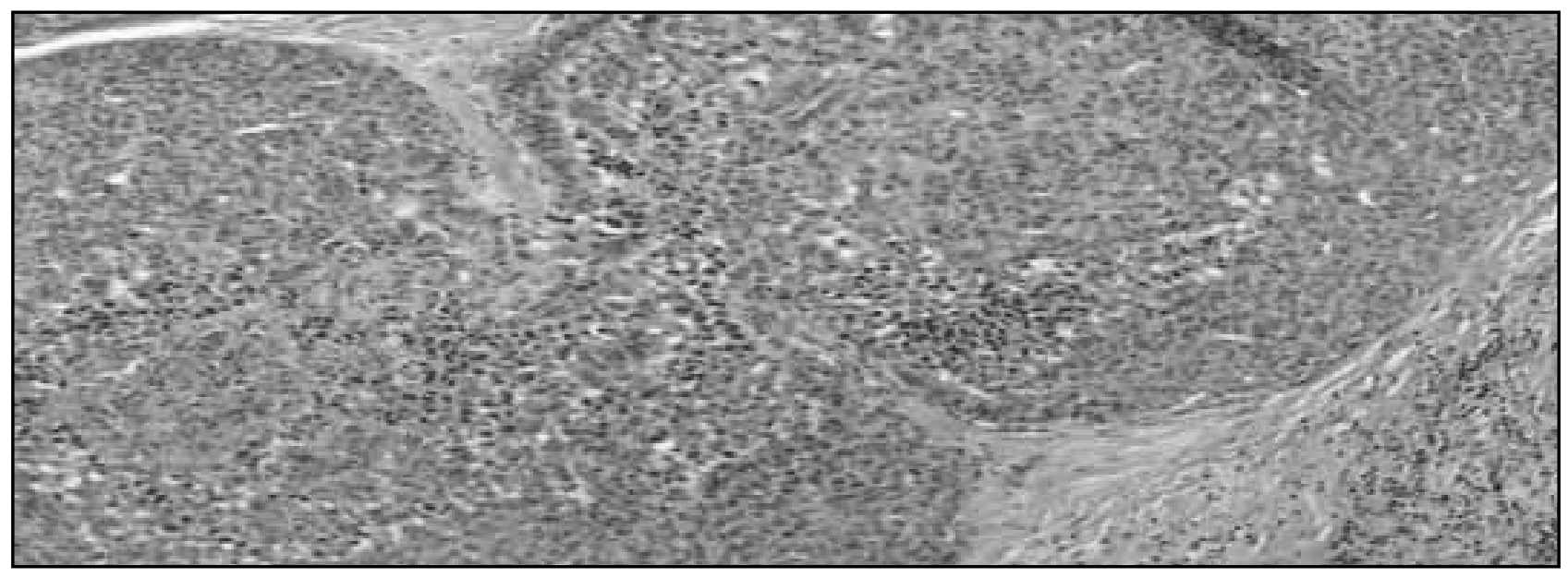

Figura 1 - TNPM tipo sólido (HE, 10x)

TNPM: tumor neuroendócrino primário de mama; HE: hematoxilina e eosina.

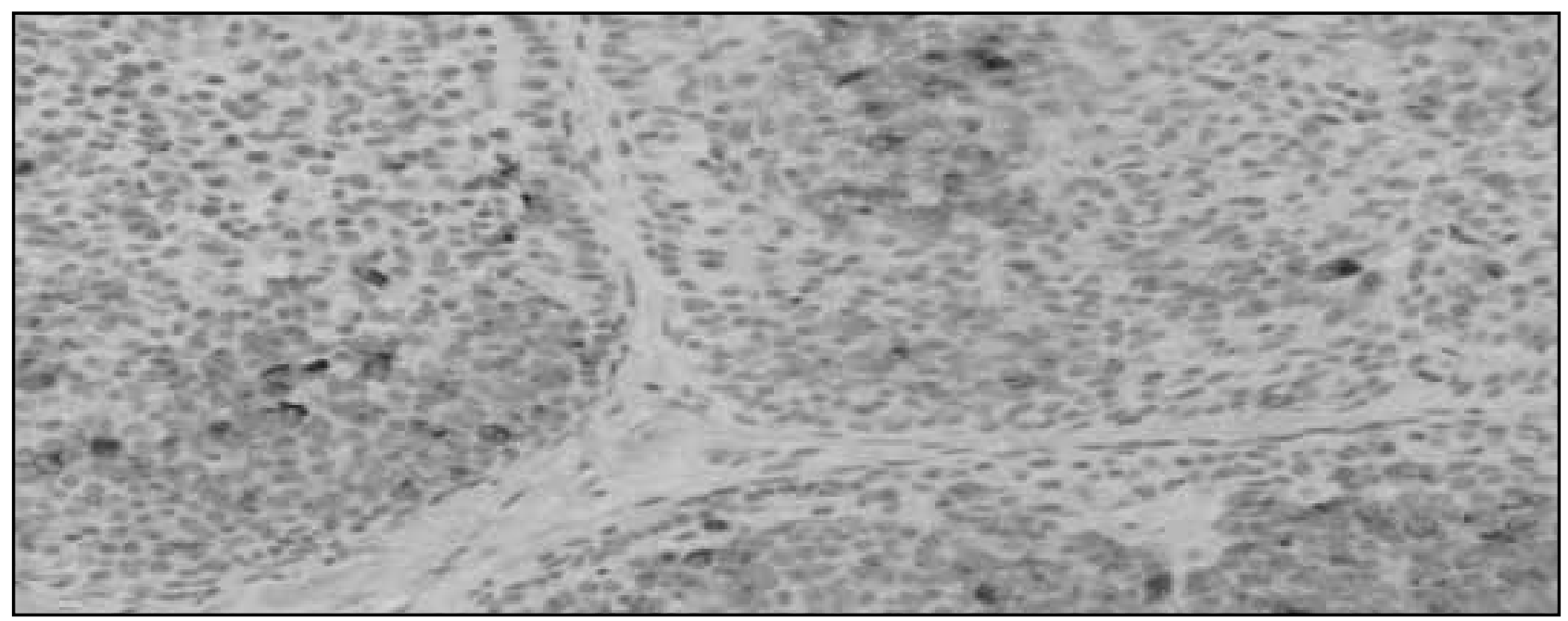

Figura 2 - Imuno-histoquímica positiva para cromogranina (20x) 


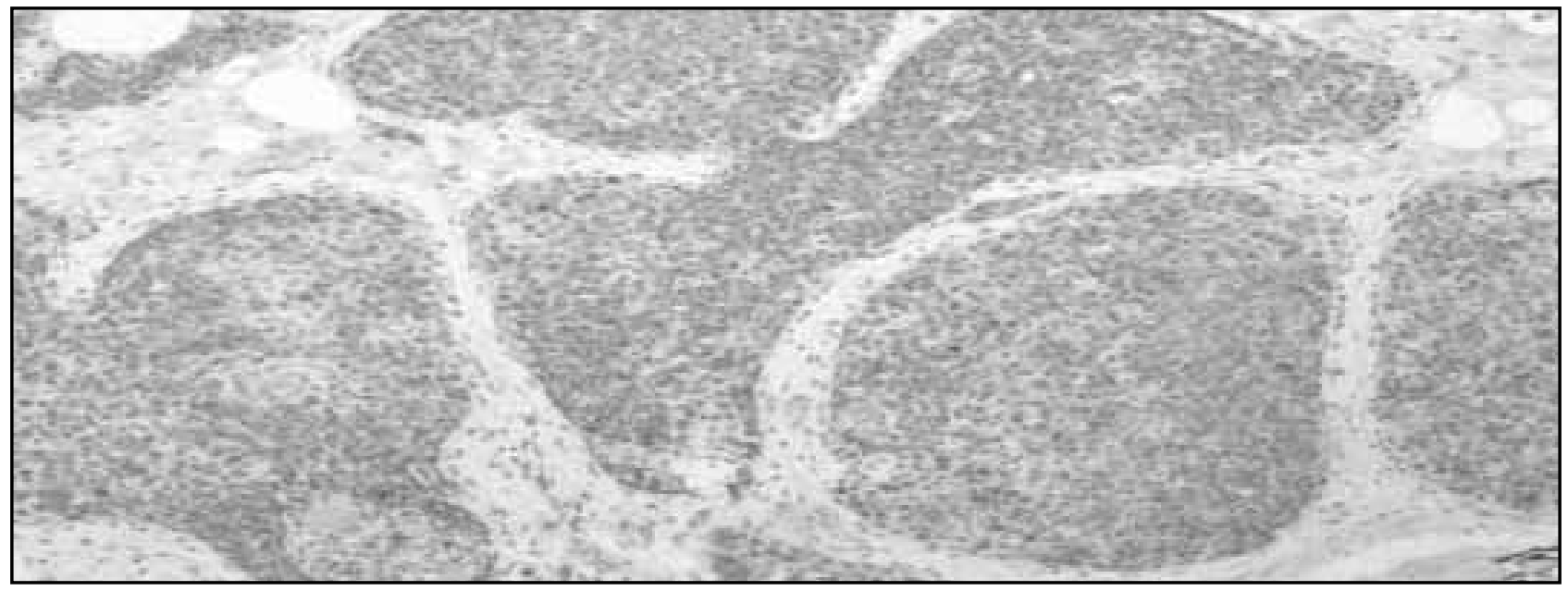

Figura 3 - Imuno-histoquímica positiva para sinaptofisina (10x)

esvaziamento axilar (um caso). A paciente do caso 2 foi a única a receber tratamento neoadjuvante contendo antraciclina e, devido à má resposta após dois ciclos, optou-se pela cirurgia. A dose de doxorrubixina utilizada nesse caso foi de $50 \mathrm{mg} / \mathrm{m}^{2}$, pouco abaixo da dose comumente utilizada $\left(60 \mathrm{mg} / \mathrm{m}^{2}\right)$ devido às comorbidades apresentadas, como hipertensão arterial, diabetes mellitus e obesidade.

Duas pacientes fizeram radioterapia adjuvante. A do primeiro relato, por apresentar tumor de $\mathrm{EC}=0$, recebeu apenas hormonioterapia, conforme protocolos atuais de tratamento. As outras pacientes apresentavam EC $=$ IIA e IIIA, respectivamente, e receberam quimioterapia adjuvante, com um esquema contendo antraciclina (caso 2) e outro não (caso 3) pelo fato de a paciente já ter sido exposta a essa substância na neoadjuvância com pouca resposta.

As três mulheres receberam hormonioterapia com tamoxifeno. Duas delas também fizeram uso de letrozol paliativo após progressão da doença. A paciente do caso 2 fez uso de letrozol por 14 meses e faleceu sem evidência de doença neoplásica; a paciente do caso 3 recebeu terceira linha de tratamento hormonal com exemestano por progressão da doença após 12 meses de uso do letrozol. Ela utilizou exemestano por quatro meses até nova progressão de doença sistêmica, quando se optou por quimioterapia, recebendo, ao todo, três linhas de quimioterapia paliativa (paclitaxel, gencitabina + cisplatina e capecitabina). Foi a única paciente que recebeu quimioterapia paliativa e que realizou radioterapias óssea e cerebral paliativas.

O seguimento dos casos evidenciou que, mesmo nas pacientes com recidiva de doença local ou a distância, houve bom controle da doença com sobrevida global de 38 e 169 meses, demonstrando comportamento menos agressivo com boa evolução clínica, a despeito de fatores adversos da doença.

Em resumo, os TNPMs são entidades ainda pouco estudadas e conhecidas por sua raridade e pequeno número de casos abrangidos pelos estudos, a maioria retrospectivo. A falta de uniformidade quanto aos tratamentos empregados e as divergências apontadas na literatura quanto a evolução e prognóstico tornam o assunto um alvo para novas pesquisas e intervenções terapêuticas. A variante tipo sólido, foco principal deste trabalho, parece se comportar de modo menos agressivo, não se diferenciando de forma significativa dos outros tumores de mama quanto aos achados clínicos, e apresenta boa correlação diagnóstica entre a morfologia e os achados imuno-histoquímicos.

\section{Referências}

1. ADEGBOlA, T.; CONNOLLY, E. C.; MORTIMER, G. Small cell neuroendocrine carcinoma of the breast: a report of three cases and review of the literature. J Clin Pathol, v. 58, p. 775-8, 2005.

2. AMERICAN JOINT COMMITTEE ON CANCER. Cancer staging manual. $7^{\text {th }}$ ed. New York: Springer, 2010. cap 32, p. 419-60.
3. CROSS, A. et al. A morphological and immunocytochemical study of a distinctive variant of ductal carcinoma in situ of the breast. Histopathology, v. 9, p. 21-37, 1985.

4. FEYRTER, F.; HARTMANN, G. On the carcinoide growth form of the carcinoma mammae, especially the carcinoma solidum (gelatinosum) mammae. Frank $Z$ Pathol, v. 73, p. 24-39, 1963. 
5. FUJIMOTO, Y. et al. A case of solid neuroendocrine carcinoma of the breast in a 40-year-old woman. Breast Cancer, v. 14, p. 250-3, 2007.

6. HONAMI, H. et al. Synchronous bilateral neuroendocrine ductal carcinoma in situ. Breast Cancer 2011. In press. Disponível em: <http://www.springerlink.com/content/ nl76834u70480478/fulltext.pdf>. Acesso em: 8 set. 2011.

7. KAWASAKI, T. et al. Neuroendocrine ductal carcinoma in situ (NE-DCIS) of the breast - comparative clinicopathological study of 20 NE-DCIS cases and 274 non-NE-DCIS cases. Histopathology, v. 53, p. 288-98, 2008.

8. KINOSHITA, S. et al. Primary small-cell neuroendocrine carcinoma of the breast: report of a case. Surg Today, v. 38, p. 734-8, 2008.

9. KITAKATA, $\mathrm{H}$. et al. A case of primary small cell carcinoma of the breast. Breast Cancer, v. 14, p. 4149, 2007.

10. LÓPEZ-BONET, E. et al. Solid neuroendocrine breast carcinomas: incidence, clinico-pathological features and immunohistochemical profiling. Oncology Reports, v. 20, p. 1369-74, 2008.

11. MAKRETSOV, N. et al. Tissue microarray analysis of neuroendocrine differentiation and its prognostic significance in breast cancer. Hum Pathol, v. 34, p. 1001-8, 2003.

12. MIREMADI, A. et al. Neuroendocrine differentiation and prognosis in breast adenocarcinoma. Histopathology, v. 40, p. 215-22, 2002.

13. NASSAR, $\mathrm{H}$. et al. Clinico-pathologic analysis of solid papillary carcinoma of the breast and associated invasive carcinomas. Am J Surg Pathol, v. 30, p. 5017, 2006.

14. ROVERA, F. et al. Neuroendocrine carcinomas of the breast. Int J Surg, v. 6, Suppl. 1, p. S113-5, 2008.
15. SAMLI, B. et al. Primary neuroendocrine small cell carcinoma of the breast. Arch Pathol Lab Med, v. 124, p. 296-308, 2000.

16. SAPINO, A. et al. Expression of apocrine differentiation markers in neuroendocrine breast carcinomas of aged women. Mod Pathol, v. 14, p. 768-76, 2001.

17. SAPINO, A. et al. Expression of the neuroendocrine phenotype in carcinomas of the breast. Semin Diagn Pathol, v. 17, p. 127-37, 2000.

18. SHIN, S. J. et al. Small cell carcinoma of the breast: a clinicopathologic and immunohistochemical study of nine patients. Am J Surg Pathol, v. 24, p. 1231-8, 2000.

19. TANG, P.; SKINNER, A. K.; HICKS, D. G. Molecular classification of breast carcinomas by immunohistochemical analysis. Diagn Mol Pathol, v. 3, n. 18, p. 125-32, 2009.

20. TAVASSOLI, F. A.; DEVILEE, P. (Ed.). WHO classification of tumours: pathology and genetics of tumours of the breast and female genital organs. Lyon, France: IARC Press, 2003. p. 32-4.

21. VAN KUMPEN, C. et al. The prognostic influence of neuroendocrine differentiation in breast cancer: results of a long-term follow-up study. Breast, v. 13, p. 32933, 2004.

22. WEI, B. et al. Invasive neuroendocrine carcinoma of the breast. Cancer, v. 116, p. 4463-73, 2010.

23. WEIGELT, B. et al. Mucinous and neuroendocrine breast carcinomas are transcriptionally distinct from invasive ductal carcinomas of no special type. Mod Pathol, v. 22, p. 1401-14, 2009.

24. YAMASAKI, T. et al. Primary small cell (oat cell) carcinoma of the breast: report of a case and review of the literature. Pathol Int, v. 50, p. 914-8, 2000.

25. ZEKIOGLU, O. et al. Neuroendocrine differentiated carcinomas of the breast: a distinct entity. Breast, v. 12, p. 251-7, 2003.

\begin{tabular}{l|l} 
& Endereço para correspondência \\
\hline Fernanda Maris Peria \\
Hospital das Clínicas da Faculdade de \\
Medicina de Ribeirão Preto \\
Setor de Oncologia Clínica (Central de \\
Quimioterapia) \\
Av. Bandeirantes, 3.900 \\
Cidade Universitária - Monte Alegre \\
CEP: 14040-030 - Ribeirão Preto-SP
\end{tabular}

